\title{
Haemopoietic regrowth after chemotherapy for acute leukaemia: an immunohistochemical study of bone marrow trephine biopsy specimens
}

\author{
B S Wilkins, A G Bostanci, M F Ryan, D B Jones
}

\begin{abstract}
Aim-To analyse haemopoietic regrowth and residual disease in bone marrow trephine biopsy specimens after treatment for acute leukaemia, using immunohistochemical staining.

Methods-Biopsy specimens before and after treatment were studied from patients diagnosed as having acute myeloid or lymphoblastic leukaemia. Specimens after treatment encompassed periods from two to 56 weeks from the start of treatment. Routine haematoxylin and eosin and Giemsa stained sections were evaluated in association with immunostained preparations. A panel of antibodies was used, which reacts with epitopes showing restricted expression dependent on the lineage or maturation stage of cells. Results were evaluated in the light of clinical, peripheral blood, and marrow aspirate findings.
\end{abstract}

Results-The speed and sequence of regrowth of haemopoietic cells were more variable than expected. Immunostaining highlighted features of dysplasia after treatment and in some cases assisted detection of residual or relapsed leukaemia. Peripheral blood and aspirate cell counts reflected accurately the amount of regrowth, but not the dysplasia, seen in biopsy samples. Delayed regrowth was associated with complex individual factors.

Conclusions-Morphological and immunohistochemical study of trephine biopsy specimens from patients treated for acute leukaemia provides information complementary to that obtained from peripheral blood and aspirated marrow. Variation in the timing and sequence of regrowth is highlighted. Immunostaining can aid in the detection of relapse or minimal residual leukaemia. The clinical relevance of dysplastic changes in biopsy specimens after treatment is uncertain, but such changes may persist for long periods.

(f Clin Pathol 1993;46:915-921)

Standard chemotherapeutic agents used to treat acute leukaemia depress normal haemopoiesis. Individual patients have cytopenias of varying severity for different lengths of time after treatment. Little is known about factors which influence the rates of regrowth of different haemopoietic cell lineages. Age, episodes of sepsis, pre-existing myelodysplasia and the nature of the chemotherapeutic agents probably all play a part, in addition to factors specific to individual patients.

Haemopoietic regrowth after chemotherapy is usually monitored by sequential examinations of peripheral blood samples. Few studies have investigated the underlying appearances in bone marrow trephine biopsy specimens, ${ }^{12}$ and little information is available on the sequential changes in the marrow as regrowth occurs. As peripheral blood findings frequently include dysplastic cellular changes which may persist for long periods, it is of interest to know whether these reflect haemopoietic stem cell or stromal cell damage sustained as a result of chemotherapy. Abnormalities in the cytology or localisation of regenerating haemopoietic cells within bone marrow could provide clues to the origin of such dysplastic changes and might provide useful variables by which to gauge the adequacy of haemopoietic reconstitution.

We and others have previously reported the use of immunohistochemical staining to demonstrate the lineage and maturation stage of haemopoietic cells within bone marrow biopsy specimens. ${ }^{34}$ In this study we used immunohistochemistry to study bone marrow trephine biopsy specimens obtained before and after treatment for acute myeloid and lymphoblastic leukaemias to assess haemopoietic regrowth and residual disease.

We have correlated our morphological findings with variables reflecting patients' clinical progress, including marrow aspirate and peripheral blood appearances, episodes of sepsis, and exposure to successive courses of chemotherapy. For most patients, at least two post-treatment biopsy specimens were available for study, and multiple biopsy specimens from some patients encompassed prolonged periods, including several episodes of disease remission and relapse.

\section{Methods}

Twenty patients, treated for acute myeloid or lymphoblastic leukaemia at Southampton University hospitals, were selected for study on the basis of availability of appropriate archival trephine biopsy material. Ages ranged from 2 to 71 years (mean 39.5 years). There were three children (ages 2, 2, and 10 years), two of whom were diagnosed as having acute lymphoblastic leukaemia (ALL) 
and one acute monoblastic leukaemia (AMLM5). Of the adult patients, 12 had acute myeloid leukaemia (AML) of various subtypes. Four had ALL, and one acute undifferentiated leukaemia (AUL). Three of the adults had myelodysplasia preceding the development of AML. Follow up ranged from two to 56 weeks after diagnosis. Chemotherapy consisted of standard regimens in current use for AML and ALL. Two patients underwent autologous bone marrow transplantation during the follow up period. Two patients were retreated after relapses at 17 and 21 weeks, respectively. They had achieved remission and completed chemotherapy for the first episode of leukaemia. Patient records were reviewed to obtain details of precise nature and timing of chemotherapy; intercurrent illnesses, particularly febrile episodes and confirmed infections; timing and findings of peripheral blood cell counts, blood films, and aspirated bone marrow; enzyme cytochemical phenotyping of leukaemic blast cells at time of diagnosis (in blood or aspirated marrow).

Seventy one biopsy specimens were suitable for study, of which 24 were taken before and 47 after treatment. The number of specimens taken after treatment for each patient ranged from one to four (most patients had two). For two patients, additional biopsy specimens were available after retreatment for relapse, as described above.

Biopsy specimens were processed routinely by fixation in $10 \%$ neutral buffered formalin, decalcification using $5 \%$ formic acid in formalin, and embedding in paraffin wax. For general assessment, sections were stained with haematoxylin and eosin. Giemsa, and a silver stain for reticulin.

Sections $5 \mu \mathrm{m}$ thick were mounted on slides coated with poly-L-lysine. After drying overnight at $37^{\circ} \mathrm{C}$, they were immunostained using a panel of antibodies selected for their reactivities with myeloid cells of different lineages and maturation stages. Details of the antibodies, their sources, and major reactivities are given in table 1. A well established streptavidin-biotin complex immunoperoxidase technique was used. ${ }^{13}$ Endogenous peroxidase activity was blocked using methanolic

Table 1 Antibodies used for immunostaining, with details of their sources and specificities

\begin{tabular}{|c|c|c|c|c|}
\hline Antibody & Specificity & Reference & Source & Reactivity in marrow \\
\hline LCA & CD45 & 5 & Dako Ltd & $\begin{array}{l}\text { Lymphoid and } \\
\text { myeloid cells }\end{array}$ \\
\hline KP1 & CD68 & 6 & D Mason, Oxford & $\begin{array}{l}\mathbf{P M} \text { and } \mathbf{M}++ \\
\text { Mono }++\end{array}$ \\
\hline NP57 & $\begin{array}{l}\text { Neutrophil } \\
\text { elastase }\end{array}$ & 7 & D Mason, Oxford & $\begin{array}{l}\mathbf{P M} \text { and } \mathbf{M}+++ \\
\mathbf{M M} \text { and } \mathbf{N P}+\end{array}$ \\
\hline LeuM1 & CD15 & 8 & Becton Dickinson & $\begin{array}{l}\mathbf{P M} \text { and } \mathbf{M}+ \\
\mathbf{M M}++\mathbf{N P}+++ \\
\mathbf{M o n o}+\text { to }++\end{array}$ \\
\hline Mac387 & Calgranulin & 9 & D Jones, Southampton & $\begin{array}{l}\mathrm{MM}++\mathrm{NP}+++ \\
\mathrm{Mono}++ \text { to }+++\end{array}$ \\
\hline BRIC 101 & $\begin{array}{l}a \text {-sialoglyco- } \\
\text { protein (a-SGP) }\end{array}$ & 10 & D Anstee, Bristol & $\begin{array}{l}\mathrm{PE} \text { and } \mathrm{EN}+ \\
\mathrm{MN}, \mathrm{LN} \text { and } \mathrm{RC}+++\end{array}$ \\
\hline Ret40f & $\begin{array}{l}\beta \text {-sialoglyco- } \\
\text { protein }(\beta \text {-SGP) }\end{array}$ & 11 & D Mason, Oxford & $\mathrm{PE}, \mathrm{N}$ and $\mathrm{RC}+++$ \\
\hline $\begin{array}{l}\text { Y2/51 } \\
\text { AA1 }\end{array}$ & $\begin{array}{l}\text { CD61 } \\
\text { Mast cell } \\
\text { tryptase }\end{array}$ & $\begin{array}{l}11 \\
12\end{array}$ & $\begin{array}{l}\text { D Mason, Oxford } \\
\text { A Walls, Southampton }\end{array}$ & $\begin{array}{l}\text { MK }- \text { to }+++ \\
\text { Mast cells }\end{array}$ \\
\hline
\end{tabular}

$\mathrm{CD}=$ cluster of differentiation; $\mathrm{PM}=$ promyelocyte; $\mathrm{M}=$ myelocyte; $\mathbf{M M}=$ metamyelocyte $\mathrm{NP}=$ neutrophil; Mono = monocyte; $\mathrm{PE}=$ pro-erythroblast; $\mathrm{EN}=$ early normoblast; $\mathrm{MN}$ mid-normoblast; $\mathrm{LN}=$ late normoblast; $\mathrm{N}=$ normoblast, all stages; $\mathrm{RC}=$ mature red blood cell; $\mathrm{MK}=$ megakaryocyte. hydrogen peroxide; trypsin digestion was performed where necessary for antigen demonstration and the final coloured product (brown) was developed using 3,3' diaminobenzidine hydrochloride (DAB) as the chromagen. Sections were lightly counterstained with Harris's haematoxylin, dehydrated, cleared, and mounted in DPX (BDH Ltd).

Sections were assessed by light microscopy, with regard to overall cellularity, distribution of granulocytic, erythroid, and megakaryocytic lineages, extent of maturation within each lineage and presence of dysplastic cytological features. For specimens taken before treatment, immunophenotypes of leukaemic infiltrates were determined by counting the number of blast cells staining positively with each antibody within a sample of 300 cells in randomly selected fields.

\section{Results}

IMMUNOPHENOTYPES OF PRETREATMENT

BIOPSY SPECIMENS

Table 2 summarises the immunophenotypes of the leukaemic infiltrates. CD45 and CD68, where positive, were expressed by all blast cells. Neutrophil elastase, CD15, and calgranulin were expressed by only a proportion of blasts cells in positive cases and their expression correlated loosely with FAB AML subtype. In particular, the presence of calgranulin was associated with monocytic differentiation (AML-M4 and M5). Unexpected reactivity with the antibody Ret 40 f (anti- $\beta$ SGP) was found in some cases of otherwise typical AML-M2 and one case of ALL. These cases were also stained using another monoclonal antibody, BRIC 4 (a gift from $\mathrm{Dr}$ D Anstee, IBGRL, Southmead Hospital, Bristol), reactive with a different epitope of SGP. Blast cells did not react with BRIC 4.

The three cases in which AML developed from myelodysplasia (all FAB subtype refractory anaemia with excess blasts (RAEB) at initial presentation) had AML phenotypes of AML-M2, M4, and M6, respectively, by the time frank leukaemia was present. The patient who developed AML-M4 had a transient phase of RAEBt in which more than $50 \%$ of blasts were erythroid: he received a single course of DAT $3+10$ at that time and immediately afterwards had marrow indicators of AML-M4.

\section{CASES OF MINIMAL RESIDUAL DISEASE}

In two cases (one AML-M2 and one AMLM4), residual leukaemic blast cells were clearly demonstrated by immunohistochemical staining of specimens (both at three weeks after treatment). In one case (AML-M4) these had not been seen with routine stains and in the other (AML-M2) they had been interpreted as equivocal, considered possibly to be regenerating normal elements.

In the case of AML-M4 expression of calgranulin and CD15 by large mononuclear cells represented a phenotype which characterised the patient's leukaemic blasts before treatment and which is found only in rare 
Table 2 Immunophenotypes of leukaemic blast cells in bone marrow biopsy specimens taken before treatment from 20 patients

\begin{tabular}{lllllll}
\hline FAB type & LCA & KP1 & NP57 & LeuM1 & Mac387 & Ret40f \\
\hline AML M1 & + & + & - & - & - & - \\
AML M1 & + & + & - & - & - & - \\
AML M2 & + & + & - & - & - & - \\
AML M2 & $(+)$ & + & - & - & - & - \\
AML M2 & + & $(+)$ & - & - & - & + \\
AML M2 & + & + & $40 \%$ & - & - & - \\
AML M2 & + & + & $20 \%$ & $15 \%$ & - & + \\
AML M2 & + & + & $(15 \%)$ & $5 \%$ & - & - \\
AML M3 & $(+)$ & + & $85 \%$ & - & - & - \\
AML M4 & + & + & - & $25 \%$ & $40 \%$ & - \\
AML M4 & + & + & - & $(35 \%)$ & $40 \%$ & - \\
AML M5 & + & + & - & $35 \%$ & $50 \%$ & - \\
AML M6 & + & $65 \%$ & - & - & - & $30 \%$ \\
ALL L1 & + & - & - & - & - & $(+)$ \\
ALL L1 & - & - & - & - & - & - \\
ALL L2 & + & - & - & - & - & - \\
ALL L2 & + & - & - & - & - & - \\
ALL L2 & - & - & - & - & - & $(+)$ \\
ALL L3 & + & - & - & - & - & - \\
AUL & + & $(+)$ & - & - & - & - \\
\hline
\end{tabular}

Figures in parentheses denote weak positive staining reaction. $-=$ no positively staining blast cells; $+=95 \%$ or more of blast cells stained positively. Percentage values (to nearest $5 \%$ ) are given for cases in which fewer than $95 \%$ of blast cells stained positively. AUL = acute undiffergiven for cases in whited leukaemia.

Cases categorised according to the French-American-British (FAB) Group classification.

mononuclear cells in normal marrow biopsy specimens. ${ }^{14}$ In both cases, however, abnormal distribution of primitive cells and lack of associated maturing forms also contributed to the diagnosis of residual leukaemia.

\section{ORDER AND TIMING OF REGROWTH}

In five cases marrow biopsy specimens showed regenerative hyperplasia affecting different combinations of erythrocytes, granulocytes, and megakaryocytes by the time of the first biopsy specimen taken after treatment (three to eight weeks after treatment). This was unrelated to age (the patients were aged $2,10,24,25$, and 44 years), diagnosis, or therapeutic regimen. Not enough subsequent specimens were available for adequate assessment of the duration of this regenerative overshoot, but normocellular marrow was found in subsequent biopsy specimens from three affected individuals two, nine, and 16 weeks later, respectively.

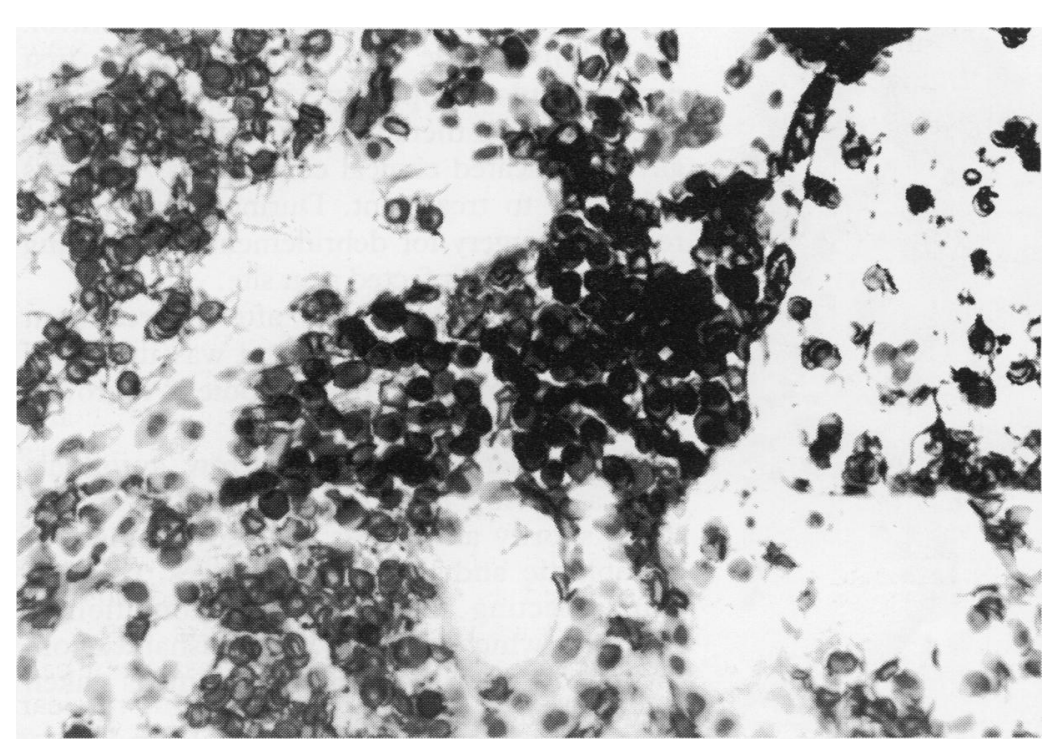

Figure 1 Erythroid cluster in which all cells seem to be at a similar stage of maturation ("synchronous cluster"). In this example most of the cells are mid-normoblasts.

Immunostained for $\beta$-SGP using the antibody Ret40f
CYTOLOGICAL DYSPLASIA AND ABNORMALITIES OF SPATIAL ORGANISATION

Cytological abnormalities were prominent in erythroid cells in 14 of 20 patients after treatment. One pattern of abnormality, seen in nine patients, was characterised by lack of the normal heterogeneity of red cell maturation stages within individual erythroid clusters, so that each cluster seemed to be composed of synchronous cells (fig 1). Such clusters appeared denser and more cohesive than normal, with sharply defined margins. Clusters of early cells coexisted in the same biopsy specimen with others composed of late cells but mixed clusters were absent. In five other cases erythropoiesis appeared megaloblastic throughout the entire biopsy specimen (fig 2).

Another abnormality of erythropoietic clusters concerned their spatial distribution. In normal marrow erythropoietic foci are not found in the immediate vicinity of trabeculae. ${ }^{15}$ In marrow biopsy specimens taken after treatment from 12 of our 20 patients, paratrabecular clusters of erythroid cells were prominent. These cases showed a sharp reduction in granulopoiesis at a time when erythropoiesis had at least partially recovered. In patients for whom later biopsy specimens were available for evaluation, the distribution of erythropoiesis returned to normal as granulopoiesis recovered.

Dysplastic cytological features within granulocytes were not seen, but a trephine biopsy specimen is not a sensitive means of evaluating such changes, especially if acid decalcification (with consequent loss of granularity) has been performed, as in our material. The most usual abnormality detected in this study was an overall reduction in the quantity of granulopoiesis, combined in some cases with a relative increase in numbers of immature forms and paucity of mature granulocytes. No striking spatial abnormalities of granulopoiesis were seen; as regrowth proceeded, early and late granulocytes repopulated their normal sites.

Megakaryocyte dysplasia was prominent in 14 of 20 cases, with abnormal clustering of cells and pronounced abnormalities of nuclear morphology in individual cells (fig 3). Irregularly segmented nuclei, mononuclear variants, micromegakaryocytes and cells with bizarre angulated nuclei were found. In patients for whom biopsy specimens taken over a long period of follow up were available, these changes persisted for many weeks or even months.

Monocytes are difficult to recognise in routinely stained biopsy specimens of normal bone marrow, but can be shown by immunostaining for calgranulin. They were absent, or present in only very low numbers, in specimens taken after treatment. Large CD68 + macrophages containing abundant phagocytosed debris were common and persisted for many weeks in some patients. Their presence was independent of the speed of regeneration of normal haemopoiesis or the presence of dysplasia.

Mast cells were shown using the antibody 


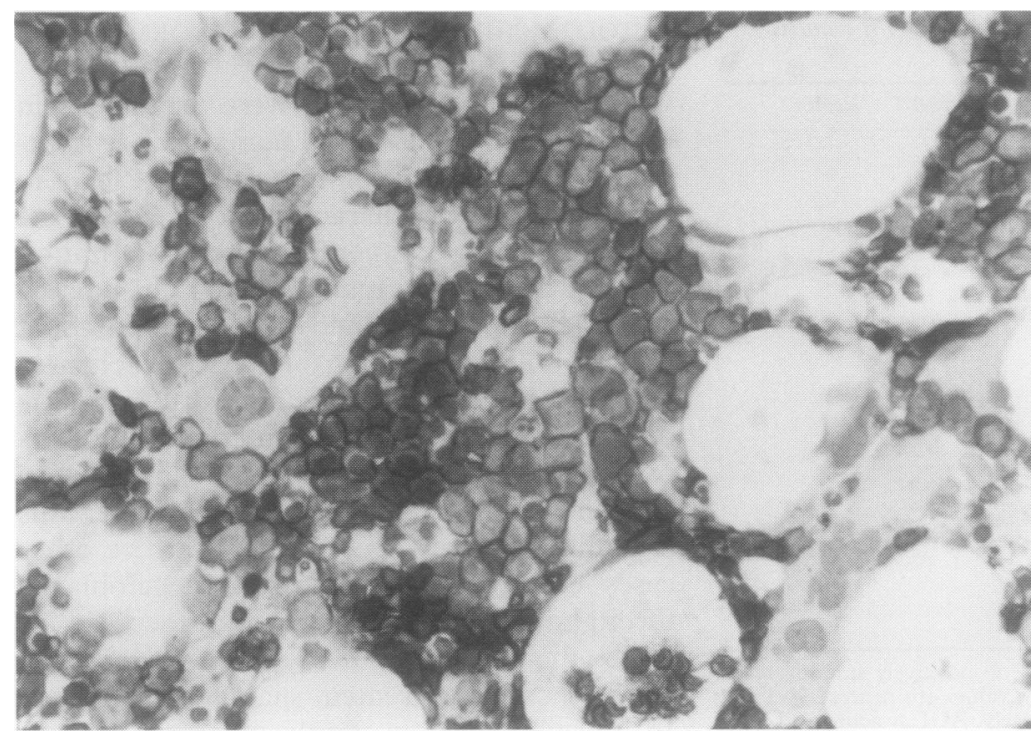

Figure 2 Megaloblast-like erythropoiesis. Irregular clusters of erythroid cells with megaloblastic cytological appearances, immunostained for a-SGP using the antibody BRIC 101.

AA1 which recognises tryptase. ${ }^{12}$ In all but four specimens they were entirely absent or were present only in small numbers (one to 10 cells per intertrabecular space). Two of the four exceptions were biopsy specimens taken before treatment from patients with ALL. Biopsy specimens taken after treatment from these patients did not contain raised mast cell numbers. The other specimens containing numerous mast cells (20 or more per intertrabecular space) were biopsy specimens taken after treatment from two patients with AML in whom the specimens taken before treatment had contained only occasional mast cells. None of these four patients showed prolonged hypoplasia after treatment. There was no correlation between the number of mast cells present and the occurrence of dysplastic changes in regenerating haemopoietic cells.

In one patient pronounced stromal fibrosis and oedema were present throughout the

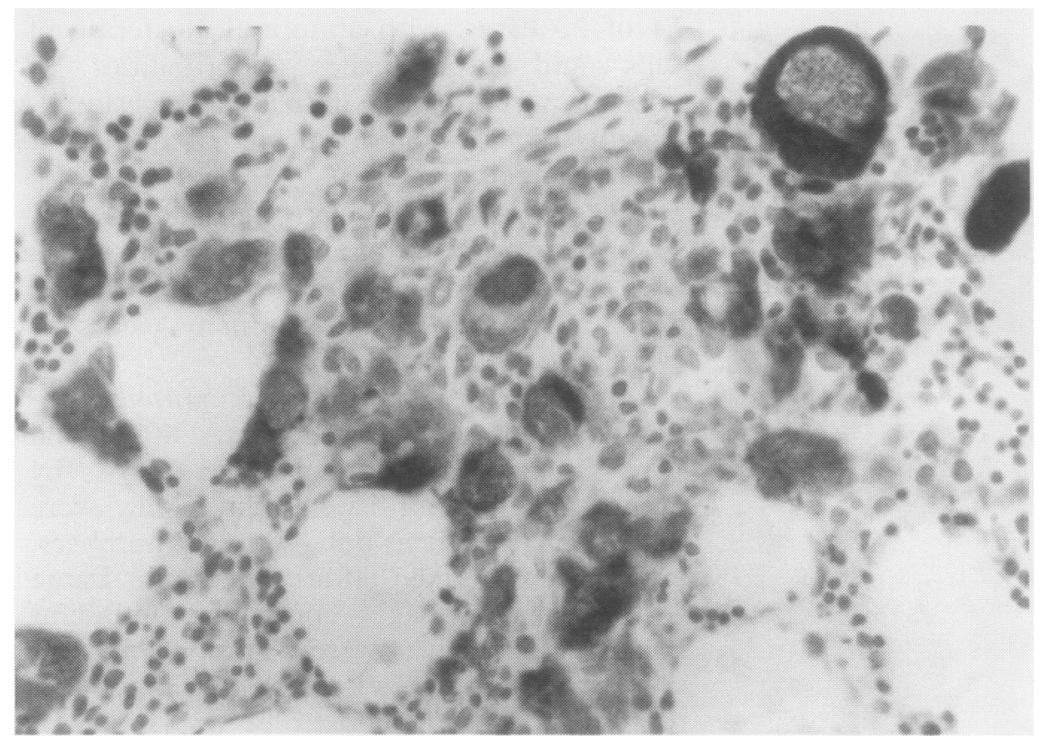

Figure 3 Megakaryocyte dysplasia highlighted by immunostaining for CD61 using the antibody Y2/51. The megakaryocytes vary widely in size and shape, with several micromegakaryocytes evident in this field. biopsy specimen four weeks after induction treatment, but good regrowth was present two weeks later, with near normal peripheral blood cell counts.

\section{CORRELATION OF BIOPSY FINDINGS WITH}

CLINICAL VARIABLES, PERIPHERAL BLOOD, AND ASPIRATE APPEARANCES

The level of erythropoiesis and granulopoiesis seen in trephine biopsy specimens correlated well with aspirate and peripheral blood findings. Megakaryocyte numbers, however, did not show a consistent relation to blood platelet counts, which returned to normal within three to four weeks, regardless of the number of megakaryocytes in the biopsy specimens.

Erythrocyte dysplasia in the biopsy specimens was accompanied by dyserythropoietic changes in aspirate smears and by anaemia. The megaloblast-like changes seen in the specimens did not correlate with megaloblastic aspirate appearances or peripheral blood macrocytosis.

Only two patients in our series had received methotrexate; one of these had normoblastic erythropoiesis. Megaloblast-like change is therefore presumed to be a nonspecific toxic effect of various chemotherapeutic agents.

There were no obvious differences between regrowth after chemotherapy for ALL rather than AML.

Dysplastic changes seen after treatment were not dependent on the presence of known myelodysplasia preceding development of leukaemia.

Regrowth seemed to be generally quicker in children and young adults than in older adults, but analysis of age in relation to time of first normocellular biopsy specimen after chemotherapy did not reveal any significant difference between age groups.

Suspected infections were treated promptly with intravenous antibiotics in all patients and there was generally no detrimental effect on marrow regrowth. One patient had a series of infections which were difficult to control. His haemopoietic regrowth was slow (first normocellular biopsy specimen at 44 weeks after induction therapy), but he had an unusually complicated clinical course. His ALL was refractory to treatment. During treatment he required surgery for debridement and grafting of a necrotising infected skin site.

Leukaemic relapse after treatment occurred in three patients and was unrelated to good or poor initial haemopoietic regrowth in this study.

Two patients received marrow autografts after achieving remission. Biopsy specimens taken shortly afterwards showed a variety of hypoplastic and dysplastic changes, presumably reflecting toxicity of the conditioning regimen, which were indistinguishable from the effects seen in biopsy specimens taken after primary treatment.

CASES OF DELAYED MARROW REGROWTH Among our series of patients, four individuals 
had exceptionally prolonged periods (more than three months) of poor haemopoietic function as judged by blood, marrow aspirate, and trephine biopsy findings. One was the patient with recurrent sepsis. The second patient had a diagnosis of AUL, subsequently revised to ALL, and her disease responded poorly to both AML and ALL therapeutic regimens; her first normocellular biopsy specimen was obtained 27 weeks after starting treatment. The third patient was a 54 year old man with AML-M4, for whom no readily identifiable cause of poor regrowth was found. His disease subsequently relapsed, after which a marrow biopsy specimen showed good regrowth with normal cellularity by six weeks. He sustained a further relapse, however, after autologous marrow transplantation 17 weeks later. The fourth patient was a 49 year old man who developed hypocellular AML-M2 on a background of myelodysplasia. Despite autologous marrow transplantation he had persistently hypoplastic marrow with prominent megakaryocyte and erythroid dysplasia up to 54 weeks from starting treatment.

\section{Discussion}

Haemopoietic regeneration after treatment for acute leukaemia is usually monitored by repeated peripheral blood and bone marrow aspirate analyses. Our results show that there is generally good correlation between the appearances in bone marrow biopsy specimens and those in concurrent blood or aspirated marrow samples over extended periods of time after induction treatment. This finding confirms the validity of blood and aspirate samples for monitoring bone marrow function during and after treatment for acute leukaemia.

A study of biopsy samples, however, offers the opportunity to evaluate aspects of the spatial organisation of haemopoietic cells, which may be of importance in determining the adequacy of reconstitution of normal haemopoietic function. Complex interactions between stromal cells, extracellular matrix molecules, growth factors and haemopoietic progenitor cells are required for normal production of blood cells. ${ }^{16}$ Abnormal spatial organisation of the bone marrow may reflect, or be a cause of, failure of this process. For example, spatial as well as cytological disturbances of haemopoietic cells are found in the primary myelodysplastic syndromes and are frequently associated with ineffective haemopoiesis. ${ }^{17}$ The use of immunohistochemistry in conjunction with spatial and cytological analysis of marrow biopsy specimens permits clear demonstration of the lineages and stages of maturation of the cells present. ${ }^{3417}$

Because of the retrospective nature of our study, the number and timing of biopsy samples from different patients were variable so that direct comparisons could not be made between all individuals. In keeping with previous studies, however, ${ }^{12}$ our results show that profound disturbances of haemopoiesis occur after treatment for AML and ALL. The availability of serial biopsy specimens from our patients allowed us to demonstrate wide variation in the speed and sequence of haemopoietic regeneration. Relatively early biopsy specimens, taken between three and eight weeks from the start of treatment, showed the existence of a transient phase of hyperplasia in a proportion of patients, the cause of which remains unexplained. Persistent hypoplasia, on the other hand, coexisted in all but one case with a complicated clinical course, albeit for different reasons in each patient.

Dysplastic changes took the form of cytological and spatial abnormalities, predominantly affecting erythroid cells and megakaryocytes. Abnormal paratrabecular distribution of erythrocytes was not necessarily associated with failure of erythroid maturation, indicating that this area of bone marrow stroma, normally occupied by early granulocytes, permits normal erythropoiesis. This suggests that marrow stromal compartmentalisation may be less rigid than cell culture derived models of specific haemopoietic microenvironments would imply. ${ }^{18}$ In our biopsy specimens repopulation of paratrabecular sites by granulocytes was accompanied by return of erythropoiesis to its more usual sites in the central areas of marrow spaces, possibly due to simple physical displacement.

The appearance of synchronous erythroid colonies in specimens taken after treatment may reflect the development of new colonies in a marrow which has been suddenly and severely depleted of the normal mixture of cells at different maturation stages. It is a feature seen in some cases of primary myelodysplasia $^{17}$ and could be the result of subtle abnormalities of interactions between stroma and developing erythroid cells. The megaloblast-like changes seen in some biopsy specimens also resembled those found in some cases of primary myelodysplasia and did not correlate with peripheral macrocytosis. In only one patient were megaloblast-like changes temporally related to treatment with a folate antagonist.

Megakaryocyte atypia was often severe and prolonged. There was no correlation between the presence of cytologically atypical megakaryocytes and abnormal peripheral blood platelet counts. Micromegakaryocytes and hypersegmented megakaryocytes, indistinguishable from those found in primary myelodysplastic $^{17}$ and myeloproliferative states, ${ }^{19}$ were seen. These abnormal appearances after chemotherapy may have been due to nuclear damage sustained by long lived, non-dividing cells in which cytoplasmic functions, including platelet production, may be relatively normal.

Granulocyte dysplastic changes were not severe, although prolonged granulocyte hypoplasia occurred in some patients. When granulopoietic cells regrew, they did so at their normal sites. This contrasts with primary myelodysplasias, in which abnormal localisation of granulopoietic cells is often a prominent feature. ${ }^{17}$ 
There have been few previous studies of trephine biopsy specimens taken during marrow regrowth after chemotherapy, 1220 and none has used immunohistochemical staining to confirm the lineages of regrowing cells. Two studies have reported biopsy findings in the early period after the start of treatment for leukaemia. ${ }^{120}$ Essentially no regrowth was present in biopsy specimens taken at day 6 and the prognostic value of the level of reduction of leukaemic cells at this stage remains uncertain. ${ }^{20} \mathrm{Up}$ to four weeks after treatment Wittels ${ }^{1}$ found little regrowth. Between four and nine weeks, erythroid and megakaryocyte numbers typically recovered more quickly than those of granulocytes.

Islam $^{2}$ has described biopsy specimens taken from 20 patients one to six weeks after chemotherapy for AML had been completed. $\mathrm{He}$ found patchy regeneration at trabecular margins and clustered centrally within intertrabecular spaces, with gradual extension from both sites to confluence. The lineages of the regenerating cells, however, were not described, nor was the presence or absence of dysplastic cytological features recorded.

Two detailed studies, one retrospective and one prospective, of trephine biopsy specimens taken after bone marrow transplantation have been reported by van den Berg and colleagues. ${ }^{21} 22$ They found many features which resemble those seen in the post-treatment marrow biopsy specimens taken after treatment and described here. In particular, they observed synchrony and megaloblastic changes in erythroid cells and clustering of megakaryocytes. They found that granulopoiesis generally recovered later than erythropoiesis, but appearances were stable and near normal one month from transplantation. The variation in order or regrowth of haemopoietic cell lineages and the noticeable persistence of some dysplastic features in our study may reflect the different composition and greater intensity of chemotherapeutic regimens used for initial treatment of AML/ALL in comparison with pre-transplant conditioning treatment.

It has been suggested that high mast cell counts may impede marrow regrowth following transplantation in cases of aplastic anaemia ${ }^{23}$ although another study found no evidence for this. ${ }^{24}$ We have already shown that immunostaining for mast cell tryptase in trephine biopsy specimens, using the monoclonal antibody AA1, reveals consistently higher numbers of mast cells than does metachromatic staining. ${ }^{25} \mathrm{We}$ were interested to see whether mast cell numbers were associated with poor regrowth in the context of acute leukaemias. High mast cell numbers were found in only two specimens taken after treatment, and showed no association with poor or dysplastic regeneration.

Stromal fibrosis has also been reported to have an adverse effect on haemopoietic recovery after marrow transplantation, ${ }^{26}$ but there was no delay in regrowth in the one patient of our series in whom fibrosis was present after induction chemotherapy. Nor were persistent abnormalities of spatial organisation or cytological atypia found in this case, suggesting that such fibrosis may be focal or transient and is not necessarily of clinical importance. Some of the cases reported by Wittels ${ }^{1}$ also developed marrow fibrosis without noticeably impaired regrowth.

Our study has also provided data of relevance to the assessment of minimal residual disease in acute leukaemia. In biopsy specimens taken as early as two or three weeks after initial chemotherapy, granulopoiesis was either completely absent or was represented by cells of all maturation stages. The quantity of granulopoiesis varied widely, but the only biopsy specimens showing focal clusters of primitive granulocytic/monocytic cells unaccompanied by later forms came from patients who had persisting leukaemic blasts in their peripheral blood or marrow aspirate samples. Although these findings are circumstantial, they suggest that clusters of primitive looking cells in biopsy specimens taken shortly after treatment should be interpreted with caution and not dismissed as normal regeneration.

Immunohistochemical characterisation of myeloid leukaemic blast cells in trephine biopsy specimens did not provide a sensitive measure of normal $v$ leukaemic regeneration in this study, reflecting the lack of detectable antigenic differences between neoplastic cells and early myeloid precursors. The rarity of monocyte precursors expressing calgranulin or CD15 in normal marrow, ${ }^{14}$ however, makes these antigens good potential targets for detection of small foci of AML-M4 or M5. A new antibody, PG-M1, reactive with an epitope of CD68 different from that recognised by KP1, has been reported recently to discriminate AML-M4 and M5 from other FAB types and to show no reactivity with early granulocytes. ${ }^{27}$ This may be of great value in future for the assessment of residual or relapsed AML-M4/M5. Detailed analysis of lymphocyte associated antigens, not a primary objective of this particular study, could similarly provide helpful information for the diagnosis of residual ALL.

1 Wittels B. Bone marrow biopsy changes following chemotherapy for acute leukemia. Am $\mathfrak{f}$ Surg Pathol 1980;4:135-42.

2 Islam A. Pattern of bone marrow regeneration following chemotherapy for acute myeloid leukemia. $f \mathrm{Med}$ 987;18:108-22.

3 Wilkins BS, Jones DB. The use of immunohistochemical techniques with fixed, decalcified, wax-embedded bone marrow trephine biopsies. Haematol Rev 1989;3: 149-63.

4 Van der Valk P, Mullink H, Huijgens PC, Tadema TM, Vos W, Meijer CJL. Immunohistochemistry in bone marrow diagnosis: value of a panel of monoclonal antibodies on routinely processed bone marrow biopsies. Am f Surg Pathol 1989;13:97-106.

5 Caldwell CW, Patterson WP, Toalson BD, Yesus YW. Surface and cytoplasmic expression of CD45 isoforms in normal and malignant myeloid cell differentiation. Am $\mathcal{F}$ Clin Pathol 1991;95:180-7.

6 Pulford KAF, Rigney EM, Micklem KJ, et al. KP1: a new monoclonal antibody that detects a monocyte/ monoclonal antibody that detects a monocyte/ macrophage associated antigen in routinely

7 Pulford KAF, Erber WN, Crick JA. Use of a monoclonal antibody against human neutrophil elastase in normal antibody against human neutrophil elastase in normal and leuka 60 .

8 Albrechtsen M, Kerr MA. Characterisation of human neutrophil glycoproteins expressing the CD15 differentiation antigen (3-fucosyl-N-acetyllactosamine). $\mathrm{Br} f$ Haematol 1989;72:312-20. 
9 Brandtzaeg P, Jones DB, Flavell DJ, Fagerhol MK Mac387 antibody and detection of formalin resistant myelomonocytic L1 antigen. $\mathcal{F}$ Clin Pathol 1988;41: myelom

10 Gardner B, Parsons SF, Merry AH, Anstee DJ. Epitopes on sialoglycoprotein alpha: evidence for heterogeneity in the molecule. Immunology 1989;68:283-9.

11 Gatter KC, Cordell JL, Turley $\mathrm{H}$, et al. The immunohistological detection of platelets, megakaryocytes and thrombi in routinely processed specimens. Histopathology 1988;13:257-67.

12 Walls AF, Jones DB, Williams JH, Church MK, Holgate ST. Immunohistochemical identification of mast cells in formaldehyde fixed tissue using monoclonal antibodies specific for tryptase. $\mathcal{F}$ Pathol 1990;162: antibodies

13 Hsu S-M, Raine L, Fanger H. Use of avidin-biotin-peroxidase complex (ABC) in immunoperoxidase techniques: a comparison between $\mathrm{ABC}$ and unlabelled antibody (PAP) procedures. F Histochem Cytochem 1981;29: 577-80.

14 Wilkins BS, Jones DB. Cell-stroma interactions in monocytopoiesis. FEMS Immunol Microbiol 1992;105:347-54.

Wilkins BS. The histology of normal haemopoiesis: bone marrow histology I. f Clin Pathol 1992;45:645-9.

16 Quesenberry PJ, McNiece IK, McGrath HE, Temeles DH, Baber GB, Deacon DH. Stromal regulation of hematopoiesis. Ann NY Acad Sci 1989;554:116-24.

17 Tricot G, de Wolf-Peeters C, Hendrickx B, Verwilghen RL. Histological findings in myelodysplastic syndromes and comparison with bone marrow smears. $B r f$ Haematol 1984;57:423-30.

18 Dexter TM, Coutinho LH, Spooncer E, et al. Stromal cells in haemopoiesis. In: Molecular Control of Haemopoiesis (Ciba Foundation Symposium 148)
Chichester: John Wiley, 1990:76-95.

19 Frisch B, Bartl R. Atlas of bone marrow pathology. In: Chronic myeloproliferative disorders Dordrecht,
Netherlands: Kluwer Academic Publishers, 1990: 97-110.

20 Roberts MM, Juttner CA, To L Bik, Kimber RJ. Bone marrow biopsy during induction chemotherapy for acute myeloid leukaemia identifies only $50 \%$ of patients with resistant disease. Leuk Res 1988;12:817-21.

21 Van den Berg H, Kluin PM, Zwaan FE, Vossen JM. Histopathology of bone marrow reconstitution after allogeneic bone marrow transplantation. Histopathology 1989;15:363-73

22 Van den Berg H, Kluin P M, Vossen J M. Early reconstitution of haematopoiesis after allogeneic bone marrow transplantation: a prospective histopathological study of bone marrow biopsy specimens. 7 Clin Pathol 1990; bone mar.

23 Naeim F, Smith GS, Gale RP, and the UCLA Bone Marrow Transplant Team. Morphologic aspects of bone marrow transplantation in patients with aplastic anemia. Hum Pathol 1978;9:295-308.

24 Sale GE, Marmont P. Marrow mast cell counts do not ( 1981;12:605-8.

25 Nock IS, Wilkins BS, Jones DB. Bone marrow mast cells revisited. F Pathol 1991;164:352A

26 Rajantie J, Sale GE, Deeg HJ, et al. Adverse effect of severe marrow fibrosis on hematologic recovery after chemoradiotherapy and allogeneic bone marrow transplantation. Blood 1986;67:1693-7.

27 Falini B, Flenghi L, Pileri S, et al. PG-M1: a new monoclonal antibody directed against a fixative-resistant epitope on the macrophage-restricted form of the CD68 molecule. Am F Pathol 1993;142:1359-62. 